\title{
Interference-Aware Radio Resource Management Framework for the 3GPP LTE Uplink with QoS Constraints*
}

\author{
Amira Afifi \\ amira.afifi@gmail.com \\ Khaled M. F. Elsayed \\ khaled@ieee.org \\ Ahmed Khattab \\ akhattab@ieee.org \\ Department of Electronics and Communications Engineering, Cairo University, Giza, Egypt 12613
}

\begin{abstract}
We propose and evaluate the performance of an integrated quality-of-service $(\mathrm{QoS})$ aware radio resource management (RRM) framework for LTE uplink access. Unlike related work, our integrated $R R M$ framework jointly takes into account 1) the class of service requirements of the different connections, 2) the constraints on the contiguity in resource block allocation due to the single-carrier frequency division multiple access (SC-FDMA) mechanism adopted for the LTE uplink, and 3) the selection of the appropriate modulation and coding scheme with full integration with the LTE uplink closed-loop fractional power control for proper power allocation. The proposed RRM framework uses closed loop power control and interference limit per cell to provide an autonomous inter-cell interference coordination scheme that is applied locally without the need of exchanging interference related information between neighboring cells. Simulation results show the ability of the proposed framework to meet the QoS of a highly loaded system with four classes of service. The interference-aware scheme also manages to limit interference and provides fair resource sharing between cell-edge and cell-center users.
\end{abstract}

Keywords- LTE; uplink scheduling; resource management; QoS; interference management; power control

\section{INTRODUCTION}

Long-Term Evolution (LTE) offers higher user data rates, reduced latency, and improved spectral efficiency [1]. One of the key technologies behind such superior LTE performance is the use of Orthogonal Frequency Division Multiplexing (OFDM) for downlink transmissions and Single Carrier - Frequency Division Multiple Access (SC-FDMA) for uplink transmissions. SC-FDMA is a Discrete Fourier Transform (DFT)-spread version of OFDM. SC-FDMA low Peak to Average Power Ratio (PAPR) saves the constrained power for the User Equipment (UE), while retaining the multipath fading resistance of OFDM and the flexibility in sub-carrier allocation. The literature of LTE uplink scheduling is not as affluent as the literature of downlink scheduling due to the complexity of the uplink scheduling optimization problem despite the importance of such a problem [2].

The typical uplink scheduling problem could be summarized as follows: Time is divided into Transmission Time Interval (TTI) of $1 \mathrm{msec}$ duration. In every TTI a decision should be made regarding which UEs will transmit, the allocation of the Physical Resource Blocks (PRBs) for the selected UEs, the transmission rate (transport format) and the maximum power the UE is allowed to use. A challenging constraint that is unique to the uplink scheduling problem is that the PRBs assignment is constrained only to contiguous subcarriers per user per TTI. Furthermore, the scheduler should also be able to take into account the QoS requirements for each user or connection while maximizing the overall throughput. All such challenges of the uplink scheduling problem should be addressed while minimizing the inter-cell interference which translates to an upper bound on the overall UE transmission power. The LTE uplink resource allocation problem with constraint on the contiguous PRB allocation is referred to as the frequency domain packet scheduling (FDPS) problem that was shown to be MAX SNP-hard even without considering other constraints such as QoS or total power constraint [2].

The existing literature of uplink RRM problem addresses the issues of PRB allocation, power allocation, and Modulation and Coding Scheme (MCS) selection separately. To the best of our knowledge, targeting throughput maximization while considering the users' QoS requirements, power control and the inter-cell interference limits have not been addressed in the literature [3-6]. In LTE systems, interference become of paramount importance because of the adoption of frequency reuse- 1 for maximizing the spectral efficiency [7]. Without proper interference management, cell-edge users suffer from excessive interference levels which eventually lead to significantly low rates and potential outages.

In this paper, we jointly tackle the problems of PRB assignment (how many) and allocation (which ones), power control and Adaptive Modulation and Coding (AMC) through MCS selection while satisfying the different classes of UE QoS requirements in one integrated framework. We present an integrated RRM framework that is composed of: 1) a time-domain scheduler that assigns priorities to the UEs based on their QoS and queue status and selects the users to be scheduled accordingly, 2) a frequency-domain scheduler that jointly tackles the PRB allocation, power control, and MCS selection problems, and 3) a closed-loop fractional power control which handles the uplink power allocation by adjusting the power control command to ensure that the overall generated cell interference is below a predefined cell interference limit. Therefore, ensuring that the proposed RRM framework respects the cell interference limit provides an autonomous Inter-Cell Interference Coordination (ICIC) scheme. ICIC is a challenging optimization problem, the solution of which requires either a central controlling entity (e.g., a controller for a group of eNB's) or an explicit coordination and information exchange between neighboring base stations. Our simulation results show that simple closed loop power control and interference limit per cell can provide an effective method for handling the ICIC problem. We also present a comprehensive set of simulation results that demonstrates the superior performance of the proposed RRM framework in terms of delay, packet drop ratio, and generated interference, at the expense of a slight decrease in the achieved throughput.

The paper is organized as follows. We discuss related work in Section II. Section III describes the system model and defines the challenges to be addressed. The proposed framework is presented in Section IV and its performance is evaluated in Section V. Section VI concludes the paper.

* This work is part of the $4 \mathrm{G}++$ project supported by the National Telecom Regulatory Authority (NTRA) of Egypt 


\section{REALTED WORK}

Existing uplink RRM problem literature addresses the issues of PRB allocation, power allocation, and Modulation and Coding Scheme (MCS) selection separately. Furthermore, the LTE uplink scheduling problem was typically studied with an assumption of infinite backlogged traffic as well as handling issues such as QoS and UE power consumption separately. In [3] the Radio Resource Management (RRM) functionality was introduced but the solution assumed fixed MCS and conventional power control scheme, the work also neglected the QoS requirements as well as channel dependent scheduling. The authors in [4] addressed the problem of channel dependent scheduling where a realistic channel estimate from the Channel State Information (CSI) extracted from the Sounding Reference Symbols (SRS) is implemented. QoS requirements such as packet loss and delay were considered in the downlink in [5-6]. To the best of our knowledge, targeting throughput maximization while considering power control and the inter-cell interference limits have not been addressed.

\section{SySTEM MODEL AND DESIGN CHALLENGES}

In this section, we describe the system model and identify the challenges that face the LTE uplink resource management and clearly define the constraints that control the problem before delving into the proposed framework details in the next Section.

\section{A. System Model}

We consider a single cell network, with one eNB and $N$ users. The cell has a total of $K$ PRB's. The users are uniformly distributed in the cell and each user has a maximum transmission power of $P_{\text {MAX. }}$. The LTE standard allows setting the UE transmit power on the Physical Uplink Shared Channel (PUSCH) through the following equation [9]

$$
P_{P U S C H}=\min \left\{\begin{array}{c}
P_{M A X}, \\
10 \log _{10}(M)+P_{o}+\alpha \cdot P L+\Delta_{M C S}+f\left(\delta_{P U S C H}\right)
\end{array}\right\}
$$

where $P_{M A X}$ is the maximum UE transmit power, $M$ is the number of allocated PRB's, $P_{o}$ is a cell specific parameter, $\alpha$ is the path loss compensation factor in the range [ $\left[\begin{array}{ll}0 & 1\end{array}\right], P L$ is the measured path loss, $\Delta_{M C S}$ is a cell/UE specific parameter related to the selected MCS scheme, and $f\left(\delta_{\mathrm{PUSCH}}\right)$ is a UE closed-loop correction value.

Channel State and Interference Awareness: Frequency domain scheduling requires the availability of the information about the channel conditions. In LTE systems, channel state information (CSI) can be extracted from the uplink Sounding Reference Symbols (SRS) transmitted by the UE and expressed in terms of a Channel-Quality Indicator (CQI) value. In our system, we assume perfect channel knowledge, i.e. we assume that the channel state is known for each user at each PRB for every TTI by the quantity and is provided by the quantity $h_{i, k}(j)$ the channel response for user user $i$ for PRB $k$ at TTI $j$ ). Furthermore we assume no delay in the CSI. The CQI value can be determined from the channel response. The Modulation and Coding Scheme (MCS) is assigned for each scheduled user on a TTI basis. The MCS assignment is based on the CQI value. Meanwhile, the uplink resource allocation is constrained to limit the overall interference of all users in the cell in any TTI to be below a predefined cell interference limit (IL) given by the value $C I L$. The interference generated is calculated by knowing the UE's transmission power and the distance estimate between each UE and the neighbor eNB's which can be estimated
TABLE I. TRAFFIC MODELS AND PARAMETERS

\begin{tabular}{|l|c|c|c|c|c|}
\hline $\begin{array}{c}\text { Traffic } \\
\text { Type }\end{array}$ & QoS & $\begin{array}{c}\text { Delay } \\
\text { msec }\end{array}$ & $\begin{array}{c}\text { Packet Error } \\
\text { Loss Ratio } \\
\text { (PELR) }\end{array}$ & $\begin{array}{c}\text { Source } \\
\text { Rate } \\
\text { kbps }\end{array}$ & $\begin{array}{c}\text { \% of } \\
\text { users }\end{array}$ \\
\hline VoIP & 4 & 100 & $10^{-6}$ & 8.375 & $30 \%$ \\
\hline FTP & 1 & 1000 & $10^{-2}$ & 680 & $30 \%$ \\
\hline Gaming & 3 & 50 & $10^{-3}$ & 8.74 & $20 \%$ \\
\hline $\begin{array}{l}\text { Video } \\
\text { Streaming }\end{array}$ & 2 & 300 & $10^{-6}$ & 64 & $20 \%$ \\
\hline
\end{tabular}

by the measurement reports sent by the UE's to its serving eNB as part of the regular scanning process with neighbor eNB's.

Traffic Model and QoS Provisioning Support: We consider four different QoS classes with four different traffic models. We adopt the LTE user traffic assignment model given in [8] in which each user has one connection. VoIP traffic is modeled as a 2-state on/off voice activity model. For video streaming, the video frame is decomposed into 8 packets with the packet size and the interarrival time between packets having a truncated Pareto distribution. The inter-arrival time between frames is deterministic at $100 \mathrm{msec}$. Interactive-gaming traffic model have the packet arrival deterministic at $40 \mathrm{msec}$ and the packet size have a Largest Extreme Value Distribution. Finally FTP traffic is modeled to have an inter-arrival time of $5 \mathrm{~ms}$ where the packet size has a uniform distribution. The four traffic classes' parameters as a result of these models are found in Table I. For QoS awareness, we assume the scheduler is capable of collecting information about the status of the queues at the UE's. Buffer Status Reports (BSR's) are signaled from the UE to the eNB to influence the scheduling decision. BSR's contain the lengths of the queues at the UE's. The BSRs are assumed to be available at each TTI for each user with no delay in the reception

\section{B. Design Challenges}

We define the interference-aware uplink resource management with QoS constraints as the problem of fulfilling the different users' QoS requirements while maximizing the overall cell throughput subject to the constraints of contiguity and maximum power constraint at each UE while preserving the overall cell interference limit. Here, we identify the different challenges that face the resource management design to help identify the constraints and define the problem.

\section{1) Fullfilling QoS requirements}

Different users have different applications and therefore different QoS requirements. During setup, every Evolved Packet Service (EPS) bearer is associated with a set of QoS parameters. These parameters include the maximum allowed delay, the maximum packet error loss rate (PELR), the bearer priority relative to other bearers and the Guaranteed Bit Rate (GBR).

\section{2) Contiguity constraint}

The use of SC-FDMA as the transmission scheme in the uplink introduces the additional constraint that all subcarriers assigned to a user must be contiguous. This constraint, combined with the constraints imposed by the UE QoS requirements, challenges the ability to maximize the system throughput by exploiting multiuser diversity. Multiuser diversity gain is obtained when the scheduler assigns a certain PRB to the user with the best channel conditions. 
3) Reducing inter-cell interefernce and meeting maximum power limits and interference limits

The orthogonality of the SC-FDMA scheme eliminates the intra-cell interference problem; however inter-cell interference still exists and is handled through uplink inter-cell interference coordination strategies [7]. To decrease the inter-cell interference experienced by cell edge UEs in the neighboring cells some restrictions are applied on the transmission power. To maximize the network throughput, the transmission power of cell edge users is restricted. In addition, each UE has a maximum transmission power limit and the cell has an overall interference limit for each cell such that the overall interference generated due to the transmissions of the UE's on neighboring cells is below CIL.

The goal of conventional power control schemes is to have all users operate at the same SINR level (also known as full compensation of path loss). Fractional power control schemes forces users with higher path loss (typically located at the cell edge) to operate at lower target SINR to decrease the interference on neighboring cells. However, equation (1) shows the coupling between power allocation, number of PRB's, and the modulation and coding scheme. Therefore, the power control problem at hand is described as follows: given a certain assigned power level and required rate, what is the number of PRB's to be assigned by the scheduler to each user to satisfy the required rate and how to load it with the appropriate MCS level. However, according to equation (1), the power level would then change according to the number of PRB's and selected MCS. Our goal is to specifically address this coupling, whereas existing state-of-the-art schemes de-couples these inter-dependent problems which leads to loss of efficiency.

\section{THE PROPOSED INTERFERENCE-AWARE SCHEDULING WITH QOS CONSTRAINTS FRAMEWORK}

In this section, we present our integrated uplink radio resource management framework. The framework solves the aforementioned correlated problems via three main stages. The first stage is a time domain scheduler that selects the set of users to be served in a given frame through assigning priorities to all users based on their QoS requirements and queue information. The second stage is a frequency domain scheduler that couples the PRB allocation, power control, and MCS selection for each of the users selected in the first stage. Finally, a closed-loop fractional power control is employed to handle the uplink power allocation to ensure a pre-defined interference upper limit.

\section{A. Time Domain Scheduling}

The time domain scheduler selects the users to be served in a given frame. It also sorts the users in the order they should be served by the FDPS. The time domain scheduler algorithm is specified as shown in Figure 1. Our proposed time domain scheduler assigns priorities to individual users, sorts them accordingly, and then selects which user to be served. The priority of the user is calculated considering two factors, the user's QoS class and the delay of the Head-of-Line (HoL) packet in the user's queue. Let $w_{\mathrm{i}}(m)$ be the HoL delay and $D_{i}^{\max }$ be the maximum allowed delay for user $i$. We follow [10] in defining the part of the priority function that considers the HoL delay, $V_{\mathrm{i}}\left(w_{\mathrm{i}}(m)\right)$, as

$$
V_{i}\left(w_{i}(m)\right)=\frac{1}{1+e^{-q_{i}\left(w_{i}(m)-D_{i}^{\max }\right)}}
$$

where $q_{i}$ is a per-class quantization constant which indicates the emergency of the traffic of user $i$ according to its required PELR (it is set to high values for classes requesting low PELR). Figure 2
1. Identify the set of active users (users with traffic in their buffers)

2. Sort the active users according to their priority as assigned by the priority function.

3. In case multiple users have the same priority, sort the equal priority users such that the user with the worse overall channel (averaged across PRB's) gets served first.

Figure 1. Pseudocode of the Time Domain Scheduler

shows the priority function for the Video and VoIP traffic classes. The priority increases as the HoL delay approaches the maximum delay limit.

Since the user only reports the buffer size in the BSR message and not the HoL delay, we redefine equation (2) to approximate the HoL delay using the BSR as

$$
V_{i}\left(s_{i}(m)\right)=\frac{1}{1+e^{-q_{i}\left(S_{i}(m)-B_{i}^{\max }\right)}}
$$

where $s_{\mathrm{i}}(m)$ is the buffer size of user $i$ at frame $m$ and $B_{i}^{\text {max }}$ is the maximum allowed buffer size for user $i$ given by:

$$
B_{i}^{\max }=\text { TrafficSourceRate }_{i} * D_{i}^{\max }
$$

Consequently, we define the priority assignment function as

$$
P_{i}=V_{i}\left(S_{i}(m)\right)+\frac{Q o S_{i}}{8}
$$

where $\mathrm{OoS}_{i}$ is the user's QoS class as shown in Table I. The QoS level is divided by eight to have values comparable to the delay part so that the effect of the delay is taken into consideration.

\section{B. Frequency Domain Scheduling}

For each selected user, the frequency domain scheduler assigns the uplink power, MCS and determines the number and location of the PRBs. Assignment is done based on the prioritized list of UE's resulting from the time-domain scheduler.

The scheme couples the PRB allocation, power control, and MCS selection together in an iterative manner. The scheme is based on the definition of a PRB group which is a set of contiguous unallocated PRB's. Initially, there is a single PRB group composed of all PRB's. When a user is allocated $k$ PRB's from PRB group $i$ which has $K_{i}$ PRB's, it would result in one the following outcomes:

1) $\mathrm{k}=K_{i}$, in this case PRB group $i$ vanishes from the set of available PRB groups.

2) $\mathrm{k}<K_{i}$, in this case PRB group $i$ shrinks in size and could result in at most two new PRB groups: a left PRB group and a right $\mathrm{PRB}$ group depending on the location and number of PRB's allocated to the user.

As the algorithm proceeds, the PRB groups keep evolving according to the allocation already made. The algorithm takes a user from the prioritized list and then makes an initial estimate of the number of required PRB's assuming the average CQI's of the user over the PRB's in the considered PRB group. Assuming the $\mathrm{UE}$ is transmitting at maximum power, the appropriate MCS is selected and the required number of PRB's to send the user pending traffic is then calculated. Consequently, the uplink power is calculated using this information and the required number of PRB's and MCS are calculated using the updated value of the power. The PRB assignment and adjusted power calculation can be iteratively repeated till convergence occurs. However, a stable 
assignment was reached after a single iteration in our simulations. Once all of the available PRB groups are checked, the user is eventually assigned to the PRB group providing it with the largest number of bits using the smallest number of PRB's. The algorithm also selects the PRB group that would result in reducing PRB fragmentation. The overall algorithm is shown in Figure 3.

\section{Uplink Power Update}

The last step of the framework is the uplink power control step to satisfy the interference limits. Two types of power control exist: open loop power control (OLPC) and closed-loop power control (CLPC). OLPC allows the UE to set its transmission power according to the available information at the receiver without coordinating with the transmitter and aims at compensating slow channel variations. On the other hand, CLPC has the eNB adjusting the UE's transmission power by sending a correction value $\delta_{\text {PUSCH }}$ known as Transmit Power Control (TPC) commands. CLPC aims to adapt to inter-cell interference changes as well as errors in path loss measurements. The adjustment function $f(t)$ could represent either an accumulative function or a current absolute value. Our framework adopts CLPC where we assume an absolute $f(t)$.

Our approach for maintaining a limit on the uplink interference generated by the cell is to map the overall CIL to individual UE interference limits. This has an important implication which is when the number of users in the system is small, each user gets on average a larger share of the interference limit, and hence, it can transmit with larger power. Consequently, larger rate per user can be attained. On the other hand, if the system has a large number of users, each user receives a smaller limit and consequently more limitation on the user power is imposed. In this work we propose three methods for dividing overall cell limit among the users depending on the target performance tradeoff.

Approach1: Equal Weights. The interference limit is equally divided among the users. This provides the simplest solution and at the same time provides fairness among the users. The downside of this approach is that the large distance of the cell center users from the cell edge is not exploited to increase the throughput, neither are the cell edge users given any advantage over the cell center users to compensate for their large path loss. Therefore while this approach provides a simple solution with good performance in terms of overall cell throughput, it does not improve the performance in terms of cell edge throughput.

Approach 2: Low Weight - High Path Loss. The second approach is to divide the interference limit in such a way that cell edge users get lower interference limits than cell center users. This approach exploits the fact that the cell center users are far enough from the neighboring eNBs such that increasing their transmission power by providing them with higher limits will have negiligible effect on the interference performance and at the same time improve the cell throughput. However, the cell edge users are now at a more of a disadvantage in this approach since they will also have a low interference limit to respect in addition to their high path loss. Therefore, the overall user satisfaction will be degraded despite the improved cell throughput.

Approach 3: Low Weight - Low Path Loss. The final approach is to divide the interference limit in such a way that cell edge users get a higher interference limit than cell center users. Since there is an overall interference limit on the cell, increasing the cell edge users transmission power should degrade the system
1. Let $U$ be the set of users to be served

2. Let $A[i]$ be the allocation matrix, $i$ is the $P R B$ index. The value of $A[i]$ is the user index assigned to the PRB.

3. Initialize A to be zeros indicating PRB is empty

4. Define PRB_Group $\mathrm{k}_{\mathrm{k}}$ as a set of empty contiguous RBs in A

for User $=1$ to number of users do

Get all available PRB Groups in A

for $\mathrm{k}=1$ to number of available PRB_Groups do

a. Calculate this user's average gain on PRB_Group

b. Get maximum power allowed to the user set by the system

c. Estimate the user's overall request from the BSR

d. Obtain the MCS from the power and calculate the average number of PRBs needed to satisfy the user request, the minimum of the calculated number of PRBs and the number of PRBs in the PRB Group is taken.

e. Calculate the closed loop power adjustment (TPC)

f. Calculate the $P_{P U S C H}$ using the outputs of steps d and e.

g. Recalculate the needed number of PRBs and the MCS using the calculated transmission power in step $\mathrm{f}$.

h. Find the PRB with the maximum gain in the PRB_Group.

i. Assign the peak PRB to the user,

j. Assign the PRBs left and right of the peak until the calculated number of PRBs is assigned.

8. end for

9. Find the PRB_Group that gives the highest number of bits to the user with the minimum number of PRBs

10. Assign this PRB Group to this user, update $\mathrm{A}[\mathrm{i}]=$ user id for assigned PRBs

11. end for

Figure 2. Pseudocode of the Frequency Domain Scheduler

performance in terms of interference only slightly. However, a kind of fairness will be provided to the users as the users with high path loss will be compensated by having a higher allowed transmission power. This approach should increase cell edge throughput and accordingly improve the user satisfaction.

Note that while in approach 1 no information about the users' locations is needed, in approach 2 and approach 3 the distance of the users from the serving eNB need to be known. In practice, the distance between the user and eNB is not known, however, it is possible to estimate the path loss between the UE and the serving eNB and use that in the weights assignment.

The interference limit for each user $i, I L_{i}$, can be calculated by:

$$
I L_{i}=\frac{C I L * w_{i}}{\sum_{k} w_{k}}
$$

where CIL is the cell interference limit in watts, and $w_{i}$ is the weight of user $i$. The weights of the users are determined according to the approach to be implemented. For approach $1, w_{i}=$ $1, \forall i$, for approach $2, w_{i} \in\{0,0.25,0.5,1,1.5,1.75,2,2.5\}$, and for approach $3, w_{i} \in\{0,0.25,0.5,1,1.5,2\}$ where $w_{i}=1$ for the user with average path loss for both approaches 2 and 3 . The values of the weights were obtained heuristically. Different step values and ranges were experimented to obtain the set of weights which give a clear distinction between the 3 approaches.

Finally, the uplink power adjustment $\delta_{\mathrm{PUSCH}}$ is calculated as shown in Figure 3. The absolute values $\delta_{\mathrm{PUSCH}}$ in $\mathrm{dB}$ are [-4 - -11 4]. To select the value to be signaled to the UE, a mapping function is designed which considers the cell interference limit (CIL). After obtaining $\delta_{\mathrm{PUSCH}}, P_{\mathrm{PUSCH}}$ is calculated using the closed loop power control equation given by (1). 


\section{Performance EVAlution}

In this section we present a comprehensive study of the performance of the proposed framework. First, we study the delay distribution of the four traffic classes as well as the overall throughput. Then the system performance is evaluated under high load conditions. Finally, we show the throughput and intercell interference for different values of SINR target and total cell interference limits. Simulations were based on the WINNER II channel model for suburban macro cell. The CLPC parameters were set to $P_{o}=-81 \mathrm{dBm} / \mathrm{Hz}$ and $\alpha=0.8$ [11]. The LTE parameters used are summarized in Table II [12].

We compare the performance of the proposed RRM framework to the First Maximum Expansion (FME) algorithm described in [13] which is one of the best known algorithms for uplink scheduling in LTE. FME does not consider the user QoS requirements into account explicitly. Although the FME allocation is not QoS-aware, we add a class-dependent behavior to FME by dropping packets exceeding their maximum delay requirement from the buffers. Basically the FME algorithm assigns the PRB to the user with highest channel gain on the PRB as long as the contiguity constraint is respected until the number of requested PRB's is satisfied or there is no more unallocated PRB's to allocate without violating contiguity constraint.

\section{A. Overall Performance: QoS Satisfaction and Aggregate \\ Throughput}

For this scenario, we generate a traffic mixture of $30 \%$ VoIP, $30 \%$ FTP, $20 \%$ interactive gaming and $20 \%$ video for 100 users. The traffic models with this distribution gave an average loadi of $22155.5 \mathrm{Kbps}$ which is around $47 \%$ of the maximum system capacity. The uplink power is adjusted to attain a per-user SINR Target of $1 \mathrm{~dB}$ ignoring interference limits.

Figure 4 shows the delay cumulative distribution functions (CDF) for all traffic classes. For the proposed RRM framework, the higher the QoS class priority the higher the slope of the curve. Furthermore, the three traffic classes with delay constraints: VoIP, gaming and video, have all the packets sent before the maximum delay is reached. Meanwhile, FTP, the best effort traffic class, experiences considerably higher delays. Note that since video has higher QoS class priority than gaming, it had high probability at low delays. On the other hand, since gaming has lower maximum delay requirement than video, the curve slope increases drastically as it approaches the maximum delay. This behavior contrasts the FME algorithm performance which disrespects the priority as depicted by the QoS requirements being not satisfied. Our results for FME show a packet drop ratio around $40 \%$ of the total number of generated packets due to missing their deadlines. Meanwhile, the proposed RRM framework had no packets dropped for the delay sensitive VoIP and video traffic, while gaming and FTP had PELR in the ranges of $10^{-3}$ and $10^{-2}$ showing that the PELR QoS parameter has been respected for all traffic classes.

\section{B. Effect of High Traffic Load on QoS Satisfaction}

We study the system with different load conditions where only two traffic models were used: VoIP and FTP. The VoIP load is fixed at $209 \mathrm{Kbps}$ and we increase the FTP load from $1100 \mathrm{Kbps}$ to $6600 \mathrm{Kbps}$. Figures 5 and 6 show the 95 percentile of delay and the throughput versus the system load, respectively. Figure 5 shows that the VoIP delay is almost constant at zero and does not exceed its limit of $50 \mathrm{~ms}$ until the FTP load increases above 4400 Kbps. This implies that the QoS parameters were respected. From this figure we can estimate the system capacity beyond which the
1. Calculate the target uplink power that generates interference equal to the interference limit. $\mathrm{P}_{\text {puschTarget }}$

2. From the received power $\mathrm{RxP}_{\text {pusch }}$ calculate transmitted power in the previous TTI TxP $\mathrm{P}_{\text {pusch }}$

3. Subtract the 2 powers and obtain the $P_{\text {difference }}=P_{\text {puschTarget }}-T x P_{\text {pusch }}$

4. Obtain the TPC command as follows

$$
\begin{array}{llll}
\text { a. If } \mathrm{P}_{\text {difference }}<=-4 & \text { then } & \text { TPC }=-4 \\
\text { b. } & \text { elseif }-4<\mathrm{P}_{\text {difference }} \leqslant-1 \text { then } & \text { TPC }=-1 \\
\text { c. elseif }-1<\mathrm{P}_{\text {difference }} \leqslant 1 \text { then } & \text { TPC }=1 \\
\text { d. } & \text { else } \quad \text { TPC }=4 & &
\end{array}
$$

Figure 3. Pseudocode of the Uplink Power Adjustment for the CLPC

TABLE II. LTE SYSTEM PARAMETERS

\begin{tabular}{|l|c|}
\hline \multicolumn{1}{|c|}{ Parameter } & Setting \\
\hline System Bandwidth & $10 \mathrm{MHz}$ \\
\hline Channel Model & C1- Suburban Macrocell \\
\hline User Speed & $60 \mathrm{~m} / \mathrm{sec}$ \\
\hline TTI & $1 \mathrm{~ms}$ \\
\hline Slot Duration & $0.5 \mathrm{~ms}$ \\
\hline Number of OFDM symbols per slot & 7 \\
\hline Noise Power & $-160 \mathrm{dBm} / \mathrm{Hz}$ \\
\hline Rx Noise Figure & $5 \mathrm{dBm}$ \\
\hline Maximum User Power & $24 \mathrm{dBm}$ \\
\hline
\end{tabular}

performance starts to degrade. Figure 6 shows that the VoIP throughput is kept constant as the FTP traffic increases. Besides, both the FTP throughput and the corresponding system throughput increase as the FTP input load increases until the system is fully loaded at FTP loads higher than $5500 \mathrm{Kbps}$.

\section{Performance with Varying Cell Interference Limit}

To study the effect of the cell interference limit, we conduct an experiment where the system load is fixed at 20 FTP users and vary the cell interference limit between $-130 \mathrm{dBm}$ and $-100 \mathrm{dBm}$. We evaluate the performance of the three individual interference limit assignment policies proposed in Section III-C. Figure 7 reports the actual generated interference versus the interference limit. As we indicated earlier, assigning low limits for cell edge users results in the smallest generated interference while assigning cell edge user high limits results in largest generated interference. Moreover, the generated interference is actually always lower than interference limits. The study is incomplete without evaluating the overall throughout and the cell edge user throughput which are reported in Figures 8 and 9, respectively. Figure 8 shows that the best overall throughput is achieved by assigning cell center users high interference limits (i.e., via approach 2). Cell-edge users throughout expressed in terms of the lowest 10-percentile user rates exhibits much better performance when cell edge users are assigned higher interference limits as shown in Figure 9. We also note the saturation behavior of the rate performance metrics for the three interference limit assigning policies. The attainable rates tend to saturate as the interference limits increases. All the values above $-110 \mathrm{dBm}$ result in almost identical performance. This means that with this level of activity and required interference limit, the system may actually allow more users in the cell without sacrificing the performance of existing users or effecting neighboring cell performance. 


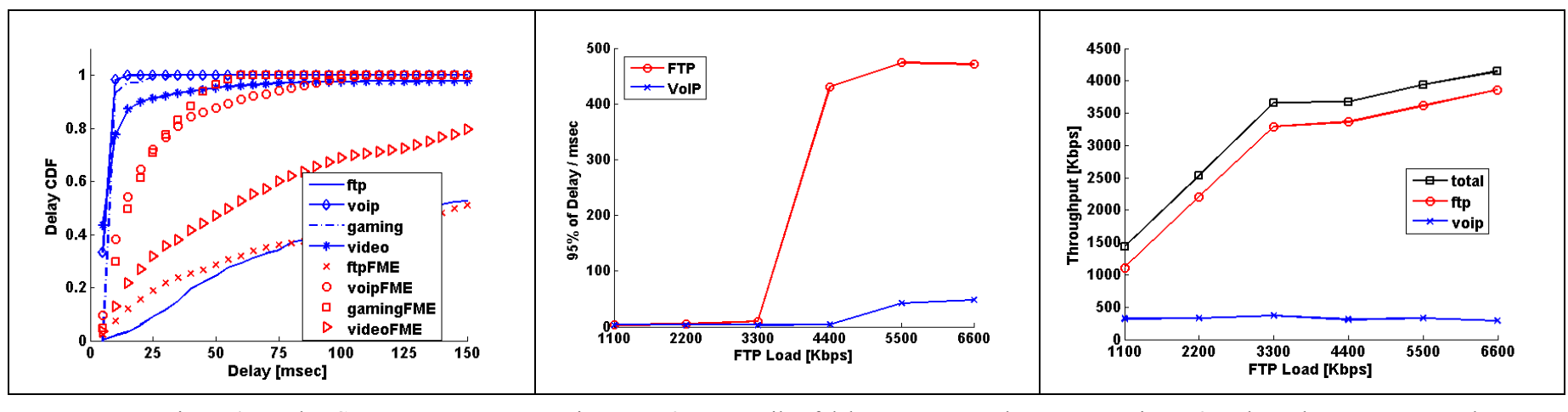

Figure 4. Delay CDF

Figure 5. 95-percentile of delay vs. FTP Load

Figure 6. Throughput vs. FTP Load

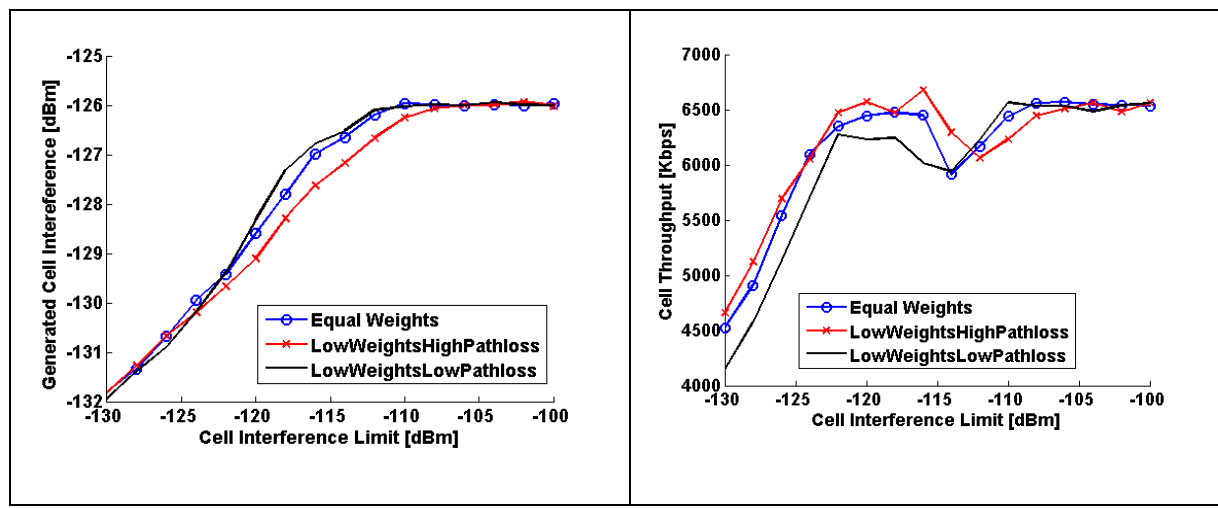

Figure 7. Generated cell interference vs. cell interference limit
Figure 8. Cell throughput vs. interference limit

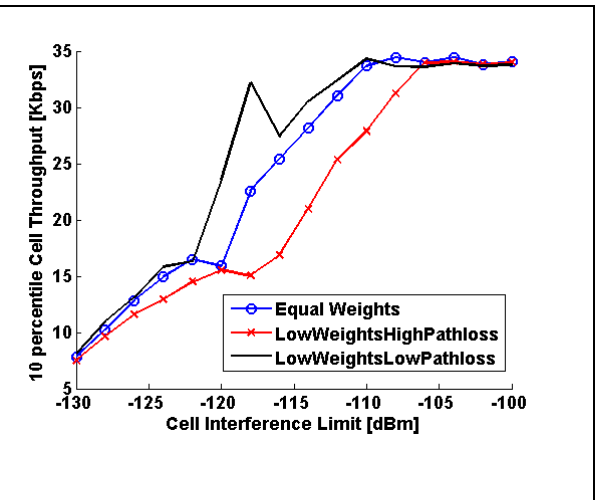

Figure 9. Cell edge throughput vs. cell interference limit

\section{CONCLUSIONS}

In this paper, we have presented a novel RRM framework which combines frequency domain channel-dependent scheduling, ICIC based power control and AMC. Unlike the related work, the framework considers the different QoS classes and their requirements in the decision making process. The conflict between the goal of channel-dependent scheduling and ICIC based power control present the tradeoff in PRBs and power allocation. We have thoroughly addressed this tradeoff in the proposed framework and analyzed its system-wide performance. By comparing with other channel dependent packet scheduling algorithms that focus on only one dimension of the problem, we have shown that the proposed RRM framework exhibits better performance in terms of delay, packet drop ratio, and generated interference, at the expense of a small decrease in throughput.

\section{REFERENCES}

[1] E. Dahlman, S. Parkvall, J. Skold, and P. Beming, "3G Evolution. HSPA and LTE for Mobile Broadband", $1^{\text {st }}$ edition Academic Press, 2007.

[2] H. Yang, F. Ren, C. Lin, and J. Zhang, "Frequency-Domain Packet Scheduling for 3GPP LTE Uplink," in Proc INFOCOM., 2010, pp. 2597-2605.

[3] F.D. Calabrese, M. Anas, C. Rosa, P.E. Mogensen, and K.I. Pedersen. Performance of a Radio Resource Allocation Algorithm for UTRAN LTE Uplink. Proceedings of IEEE Vehicular Technology Conference (VTC) 2007 Spring, April 2007.

[4] F. D. Calabrese, P. H. Michaelsen, C. Rosa, M. Anas, C. U. Castellanos, D. L. Villa, K. I. Pedersen, and P. E. Mogensen, "Search-Tree based Uplink Channel Aware Packet Scheduling for UTRAN LTE," in
Proceedings of the 67th IEEE Vehicular Technology Conference (VTC), Singapore, May 2008, pp. 1949-1953.

[5] G. Monghal, K. I. Pederson, I. Z. Kovacs and P. E. Mogensen, "QoS Oriented Time and Frequency Domain Packet Schedulers for The UTRAN Long Term Evolution" IEEE 67thVTC, May 2008.

[6] K. Sandrasegaran, H. A. M. Ramli, and R. Basukala, "Delay-Prioritized Scheduling (DPS) for Real Time Traffic in 3GPP LTE System" in Proceedings of the WCNC 2010.

[7] G. Boudreau, J. Panicker, N. Guo, R. Chang, N. Wang, and S. Vrzic, "Interefernce Coordination and Cancellation for 4G Networks", IEEE Communication Magazine, April 2009.

[8] Orange, China Mobile, KPN, NTT DoCoMo, Sprint, T-Mobile, Vodafone, Telecom Italia, "LTE physical layer framework for performance verification,” R1-070674, 3GPP TSG-RAN1\#48, St. Louis, Mich, February, 2007.

[9] Technical Specification Group Radio Access Network, "Evolved universal terrestrial radio access (E-UTRA); physical layer procedures," 3rd Generation Partnership Project (3GPP), Tech. Rep. TS 36.213, Mar. 2009.

[10] S. M. Shukry, K. Elsayed, A. Elmoghazy, and A. Nassar "Adaptive fractional frequeny reuse (AFFR) scheme for multi-cell IEEE 802.16e systems," Proceeding of HONET'09, 2009.

[11] B. Muhammad, "Closed Loop Power Control for LTE Uplink", Thesis presented as part of Degree of Master of Science in Electrical Engineering, Blekinge Institute of Technology, November 2008.

[12] L. Hentila, P. Kyosti, M. Kaske, M. Narandzic, and M. Alatossava, MATLAB implementation of the WINNER phase II channel model ver1.1, Dec. 2007.

[13] L. Ruiz de Temiño, G. Berardinelli, S. Frattasi, and P.E. Mogensen, "Channel-aware scheduling algorithms for SC-FDMA in LTE uplink", in Proc. PIMRC, 2008, pp.1-6. 